\title{
The Myth of Idiopathic Granulomatous Mastitis.
}

Adel Mohamed Khalaf, ${ }^{1}$ MD,Gamal Galal Al-Shimy, ${ }^{1}$ MD, Abd Al-Hameed Hefny, ${ }^{1}$ MD.

* Corresponding Author:

Adel Mohamed Khalaf

dr.adel_khalaf@hotmail.com

\section{Received for publication October 20, 2020; Accepted December 20, 2020; Published online}

December 20, 2020.

Copyright 2020 The Authors published by Al-Azhar University, Faculty of Medicine, Cairo, Egypt. All rights reserved. This an open-access article distributed under the legal terms, where it is permissible to download and share the work provided it is properly cited. The work cannot be changed in any way or used commercially.

doi: 10.21608/aimj.2020.46076.1335.

${ }^{I}$ Department of general surgery, Faculty of medicine, Al-Azhar University, Assuit, Egypt.

${ }^{2}$ Department of general surgery, Faculty of medicine, Al-Azhar University, Damietta, Egypt

\begin{abstract}
Background: Idiopathic granulomatous mastitis (IGM) is a benign rare inflammatory pseudotumor. The final diagnosis is made by biopsy and histopathology.

Aim of the study: to present clinical features of IGM and to evaluate the results of treatments.

Patient and Methods: Ten female cases who full-field required clinical and histologic criteria of IGM were enrolled in the study. Demographic data, clinical presentation, medication history, and radiologic results are presented. To confirm the difference in clinical course between IGM patients and other inflammatory breast disease patients, a review of the clinical and pathological data was also performed for patients with symptoms similar to these IGM patients.

Results: The Median age was 28 years (range 22-42). Nine patients had no recurrence during the follow-up period. Bacterial cultures and pathological tissue diagnosis were performed. After median follow-up of 15 months (range 6-24), one (10\%) patient developed disease recurrence, as confirmed by repeat histological examination.

Conclusion: According to our findings, histopathology of the disease is fundamental for correct diagnosis. Corticosteroid therapy as a sole therapeutic method (prednisolone) proved to be an applicable and effective choice in the control of idiopathic granulomatous mastitis by decreasing inflammation. Surgical management should be avoided unless all medical treatment options have been exhausted. IGM is a chronic breast inflammatory disease with a recurrence incidence of $10 \%$ in the present study, especially if there is history of cigarette smoke exposure and/or isolation of Corynebacterium from tissue/pus sample.
\end{abstract}

Keywords: Breast; Granulomatous; Mastitis; Idiopathic; Diagnosis.

Disclosure: The authors have no financial interest to declare in relation to the content of this article. The Article Processing Charge was paid for by the authors.

Authorship: All authors have a substantial contribution to the article.

\section{INTRODUCTION}

Since the first pathological announcement of idiopathic granulomatous mastitis [IGM] as a benign pathology ${ }^{1}$, many patients all over the globe have been reported. Idiopathic granulomatous mastitis [IGM] was rare diagnostic item with approximately $2 / 100,000$ incidence $[0.35 \%]$ in the States. ${ }^{2}$ Majority of diagnosed patients in the States are non-whites. Incidence in most European countries is less. ${ }^{3}$ Idiopathic granulomatous mastitis [IGM] is an uncommon inflammatory (benign) pathology that occurs in mothers of childbearing period with recent breast-feeding history. The rare nature of this disease associated with lacking of important data. The exact etiology of idiopathic granulomatous mastitis [IGM] is still unknown thus no agreement existed on its management. ${ }^{4}$ Exact etiology and pathophysiology still unknown. There is phlegmasia as a sequence to trauma hormonal or metabolic or autoimmune reaction and Corynebacterium contamination. All these factors have been accused. ${ }^{5}$
Presentations includes mass and inflammatory manifestations. Presentations are similar to bacterial mastitis and carcinoma (inflammatory). There is long history of the disease. Final diagnosis is achieved by open biopsy and histopathological examination. Biopsy report non-specific granuloma with scattered epithelioid cells giant cells (multi-nucleated) and plasma cells. Ultrasonogram, mammogram, and MRI (magnetic resonance imaging) are inconclusive. Mammography and ultrasonography are important to exclude other breast disorders .

Management of patients with idiopathic granulomatous mastitis [IGM] remains a great challenge for the medical professions and patients who suffered an exhausting course of the disease with a negative impact on the quality of life itself. Other mastitis etiologies and specifically malignancy should be excluded before the diagnosis of idiopathic granulomatous mastitis [IGM] considered. Due to data 
lack especially randomized controlled trials, idiopathic granulomatous mastitis [IGM] management is debatable. Most authors in developed world use regimens as combination of corticosteroids and antibiotics, with subsequent continuous low dose steroid, immunosuppressant. Surgery should be considered in breasts with persistent disease. ${ }^{6}$

A lot of information still needed to specify the best line of treatment. Therapy with immunomodulators is not yet brought to light. Fulfillment of a registry for collection of precious data on this uncommon change is absolutely recommended. Granulomatous mastitis may be the end-result of many specific infections of the breast such as tuberculosis, brucellosis, leprosy, fungal and parasitic infections. In many cases, the actual cause remains unknown (idiopathic). Granulomatous reaction may also be detected in the vicinity of breast carcinomas. ${ }^{7}$

We present our series of 10 patients with idiopathic granulomatous mastitis [IGM] who treated as their clinical manifestations. Cases presented with abscess formation operated upon under general anesthesia by deep multidirectional drainage. These patients were treated medically (no corticosteroids), except two patients. Our results were evaluated as regard outcome of treatment in comparison with corticosteroid regimes discussed in the literature.

\section{PATIENT AND METHODS}

Women with biopsy proven idiopathic granulomatous mastitis [IGM] were included in the study. Patients who fit the histologic criteria of idiopathic granulomatous mastitis [IGM] were identified (noncaseating granulomas centered on lobules, with or without associated micro-abscesses). Women were enrolled in the current study after exclusion of all other possible causes of granuloma formation. Diagnosis was confirmed by Tru-cut needle or excisional biopsies, or from materials taken from the wall of the abscess during drainage. Slides were stained with hematoxylin-eosin and more specific stains (Gram, Ziehl-Neelsen, and periodic acidSchiff). Pathological slides were revised by a senior pathologist. Totally, 10 specimens of idiopathic granulomatous mastitis [IGM] were encountered .

Comparison of the clinical course between idiopathic granulomatous mastitis [IGM] breasts and other inflammatory diseases of the breast performed. Review of the clinical and pathological data was performed for cases with manifestations similar to idiopathic granulomatous mastitis [IGM] patients, according to the first proposed diagnosis "mastitis" or "breast abscess." Cases diagnosed as tuberculous mastitis were excluded from the current study. Standard protocol for management was proposed to all patients after Tru-cut needle biopsy proved idiopathic granulomatous mastitis [IGM]. All patients with IGM associated pathogens (e.g. Mycobacterium tuberculosis, Histoplasmosis, Blastomycosis, etc.) were excluded from the study. Patients with hepatitis
B, or C (either infected or carriers) were excluded before commencing steroid treatment. Multidisciplinary approach of management (breast surgery, microbiology, pathology, radiology, and rheumatology) was conducted. Drainage of collection (percutaneous or open drainage) was performed .

Antibiotics were prescribed when superimposed infection is suspected or tissue / pus culture was positive for bacterial growth. Steroid therapy was commenced $(20 \mathrm{mg}$ two times daily for 4 weeks. Steroid dose was tapered over subsequent 4 weeks). Reassessment by clinical review and breast imaging was performed. Patients with complete resolution were continued clinical and radiological monitoring. Patients with residual breast mass offered surgical excision. Reassessment of all patients was conducted by clinical review and breast imaging. Patients with clinical and/ or radiological relapse were reassessed by multidisciplinary team, repeated steroids, antibiotics, and drainage if indicated. Addition of immunomodulatory agents (e.g. Azathioprine) was tried. Patients with evidence of resolution and no recurrence were continue clinical and radiological monitoring.

According to the clinical presentation management plans were designed. Patients with pus collection were treated with surgical drainage with antibiotics and oral steroids after hepatitis B and C exclusion. Antibiotics also prescribed if secondary bacterial infection was evident, according to results of culture and sensitivity of the organism. Clinical and radiological monitoring was continued with frequent breast ultrasound. Excision of residual masses was performed following conservative management.

Complete resolution was achieved by disappearance of all clinical manifestations plus absence of all inflammatory signs by ultrasound scan. Patients were followed up and observed for recurrence. Demographic features, clinical manifestations and specific characteristics of idiopathic granulomatous mastitis [IGM], microbiology, and pathology reports, were analysed .

Data of the patient's presentation, histopathological results, treatment, and outcome were examined by revision of their medical files. Follow-up data were harvested from clinical reviews. Symptoms, their severity, and duration were also documented. Collected data were analyzed and all parameters were evaluated retrospectively. Potential risk factors were analyzed by univariate and multivariate logistic regression.

\section{RESULTS}

Ten patients were diagnosed and treated for idiopathic granulomatous mastitis [IGM] during the period of the study. Age (median) was 28 years (ranged from 22 to 42). Size of the mass and/or abscess (median) was 52 $\mathrm{mm}$ (ranged from 20 to 110). Six patients presented with mass [painful] $(\mathrm{n}=6,60 \%)$. Other patients presented with mass [painless] $(\mathrm{n}=1,10 \%)$, abscess $(\mathrm{n}=3,30 \%)$ (Table 1). One patient $(10 \%)$ was 
cigarette heavy smoker. One patient was diabetic. Medical history of the other eight patients was unremarkable.

\begin{tabular}{|l|l|}
\hline \multicolumn{1}{|c|}{ Presentation } & \multicolumn{1}{c|}{ No (\%) } \\
\hline Symptoms and signs: & \\
\hline Mass & $7(70 \%)$ \\
Ulcer & $0(0 \%)$ \\
Mastitis (abscess) & $3(30 \%)$ \\
Fistula & $1(10 \%)$ \\
Pain & $9(90 \%)$ \\
\hline No of mass lesions: & \\
\hline 0 & $0(0 \%)$ \\
1 & $8(80 \%)$ \\
2 & $2(20 \%)$ \\
\hline Lesion & \\
\hline location: Side Rt & $6(60 \%)$ \\
location: Side Lt & $4(40 \%)$ \\
Retroareolar & $1(10 \%)$ \\
UIQ & $1(10 \%)$ \\
UOQ & $3(30 \%)$ \\
LIQ & $2(20 \%)$ \\
LOQ & $3(30 \%)$ \\
\hline Birads & \\
\hline 2 & $1(10 \%)$ \\
3 & $3(30 \%)$ \\
4 & $5(50 \%)$ \\
5 & $1(10 \%)$ \\
\hline &
\end{tabular}

Table 1: Clinical presentation of 10 patients diagnosed with IGM.

Mammographic features ranged from sound architecture in patients with huge and dense breasts to features of benign or even malignant masses and asymmetric focal density. prevalent mammographic features of the lesion was increased density (asymmetrical), which is non characteristic in this entity.

Ultrasonographic features of the disease were also varied and assumed to be related to the histological features. Ultrasonographic findings included masslike shadows, tubular and nodular hypoechoic appearance. Focal decreased parenchymal echogenicity were also encountered with acoustic shadowing.

The most frequent finding on MRI was asymmetrical signal intensity (focal or diffuse) without significant mass effect (regions tends to be hypo-intense-T1), and $\mathrm{T} 2$ regions tend to be hyperintense). Also, nodular lesions were seen.

Magnetic resonance imaging (Dynamic contrastenhanced) showed mass-like pattern, nodular enhancement and/or ring-like enhancement. Time- intensity curves were different from lesion to lesion and from patient to patient.

Pathology: Immunologic theory assumed as possible causes. Vasculitis absence and heavy lymphocytic infiltration in the biopsies favored against this theory. Diagnosis of idiopathic granulomatous mastitis [IGM] was based on exclusion of other diseases. Diagnosis was dependent on demonstration of a specific histopathological patterns with exclusion of specific granulomas such as: tuberculosis (Fig. 1), sarcoid reaction, granuloma with polyangiitis, infections caused by fungi, and other non-malignant inflammatory patterns associated with carcinoma. During pathological examination, idiopathic granulomatous mastitis [IGM] manifested as noncaseating, non-vasculitic granulomatous reaction in lobules (Figs. 2-4). In advanced cases, end-stage features as abscess, fat necrosis, and fibrosis disturb the normal lobular architecture.

Bacterial cultures were performed in all patients, in which $6(60 \%)$ was -ve for bacterial growth. Among 4 samples with +ve growth, Corynebacterium infection was proved from culture in two cases. In addition, one patient had Staphylococci, and one had Streptococci isolated from their culture and sensitivity.

Five patients received medical treatment (oral corticosteroid) alone $(\mathrm{n}=5,50 \%)$, four $(40 \%)$ patients received antibiotics plus oral corticosteroid. and one (10\%) patient did not receive either steroid or antibiotic. Surgery (drainage) was performed in 3 cases. Two patients (20\%) underwent abscess drainage. One patient $(10 \%)$ received percutaneous drainage. Three patients $(30 \%)$ underwent excision of the remaining breast swelling.

After follow-up of 15 months (median, ranged from 6 to 24 ), only one patient (10\%) developed recurrence. Confirmed by repeated histopathological evaluation. Univariate analysis for prognostic factors (age, lesion size, previous health condition, smoking, and results of bacterial culture and sensitivity. Isolation of Corynebacterium $(\mathrm{p}=0.0001)$, cigarette smoking $(\mathrm{p}<$ 0.001 ), and presence of abscess on first examination $(\mathrm{p}<0.02)$ were statistically significant (as shown in table 2). Otherwise, statistically significant relation between different treatments and recurrence was not found.

Multivariate analysis was performed for recurrence (using Cox proportional hazards model). Initial model included factors proved to be positive from univariate analysis (isolation of Corynebacterium, smoking, and abscess formation on first presentation). Actually, Corynebacterium +ve lesions and smoking were significant factors of recurrence in multivariate analysis [(OR) 30.138, p = 0.040, and OR 44.825, p = 0.004 , respectively]. 


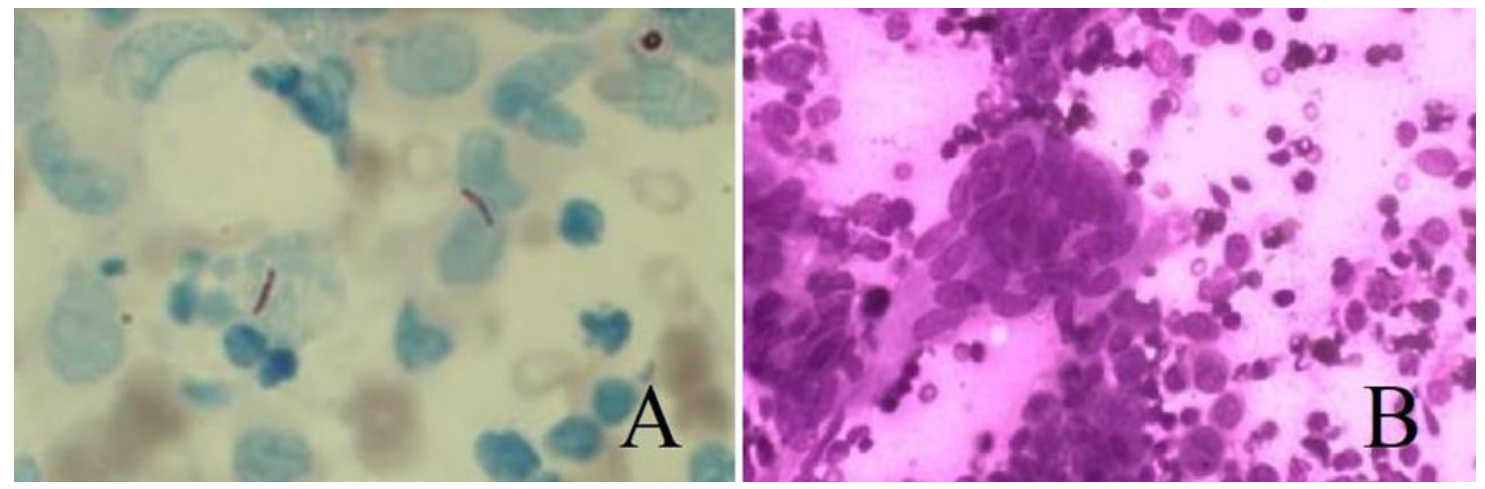

Fig. 1: (A): ZN stain shows AFB in background of polymorphs and epithelioid histiocytes with (B): multinucleated giant cells.

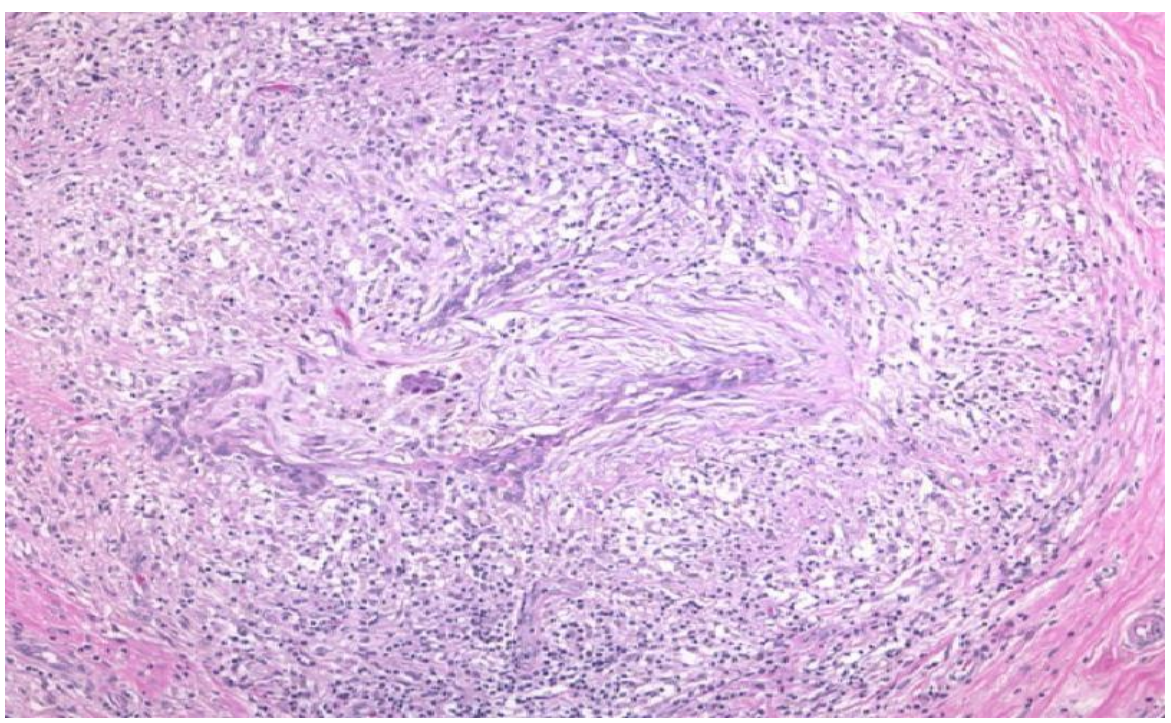

Fig. 2: Natural architecture of the breast has been destroyed and a condensation of inflammatory cellular infiltrate surrounds the residual distorted ducts.

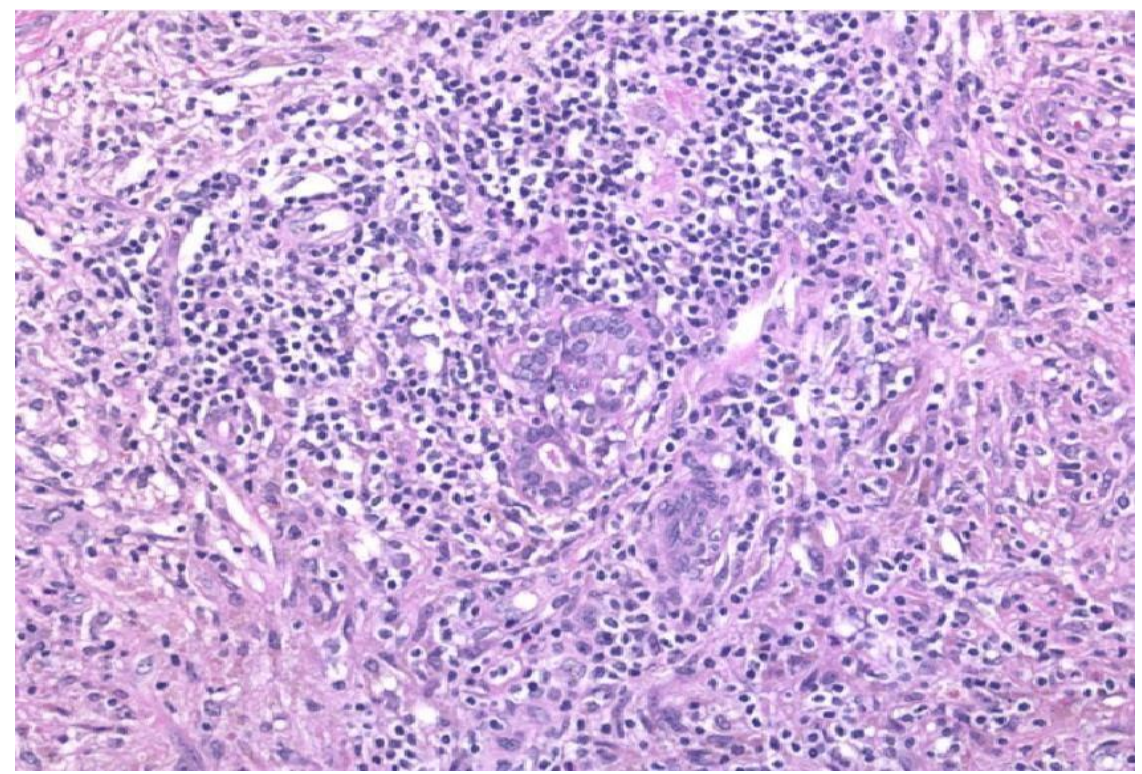

Fig. 3: Cell-mediated inflammatory response of granulomatous mastitis, consisted of epithelioid histiocytes, Langhans' giant cells, lymphocytes, plasma cells, and a few eosinophils. Areas of fat necrosis and even microabscesses may be seen. 


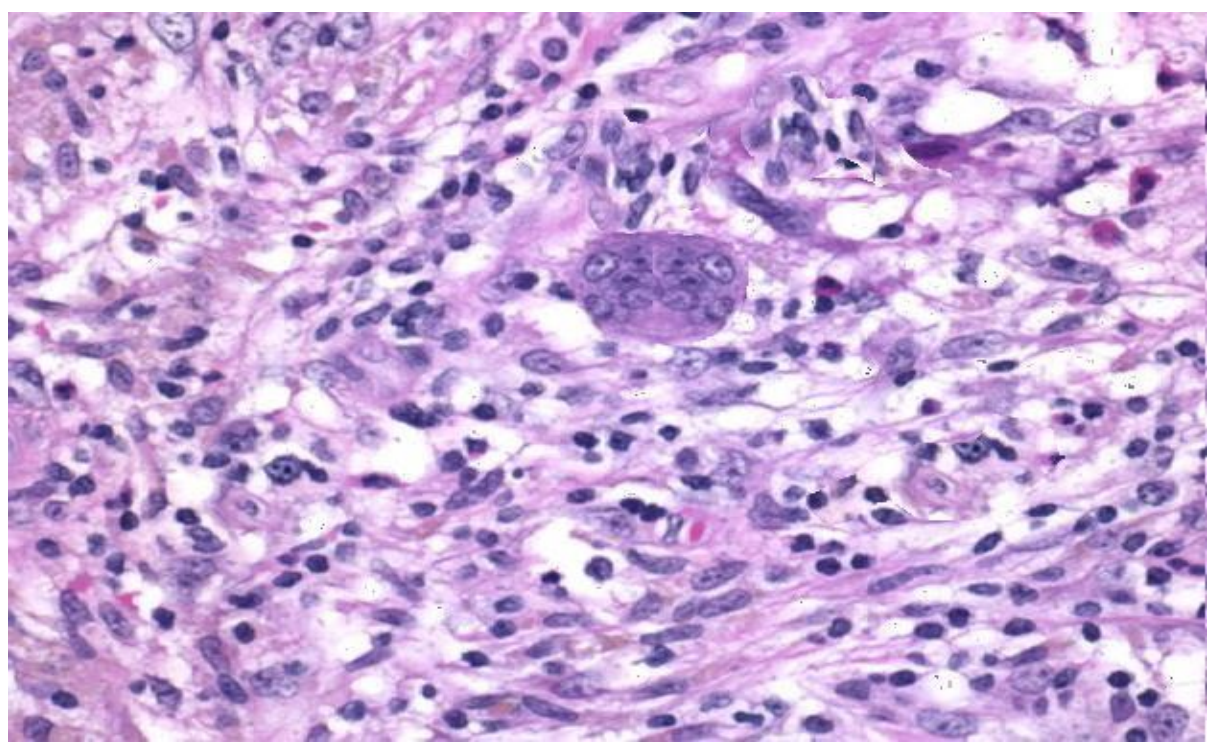

Fig. 4: Center of a granuloma in granulomatous mastitis show a Langhans' giant cell surrounded by epithelioid histiocytes, lymphocytes, and a few eosinophils (high-power view).

\begin{tabular}{|c|c|c|c|}
\hline & No recurrence & Recurrence & P value \\
\hline Age $<30 y$ & 3 & 0 & 0.8 \\
\hline$>30 y$ & 6 & 1 & \\
\hline Size $<40 \mathrm{~mm}$ & 4 & 0 & 0.06 \\
\hline$>40 \mathrm{~mm}$ & 5 & 1 & \\
\hline Cigarette smoke exposure No & 8 & 1 & 0.001 \\
\hline Yes & 1 & 0 & \\
\hline Diabetes mellitus No & 9 & 0 & 0.156 \\
\hline Yes & 0 & 1 & \\
\hline Presentation Abscess & 2 & 1 & 0.020 \\
\hline No abscess & 7 & 0 & \\
\hline Isolation of Corynebacterium +ve & 1 & 1 & 0.0001 \\
\hline -ve & 8 & 0 & \\
\hline
\end{tabular}

Table 2. Clinical and microbiological correlation in patients with or without disease recurrence.

\section{DISCUSSION}

Idiopathic granulomatous mastitis [IGM], as its name referee, is a chronic non specific inflammatory disease of the mammary gland with granulomatous tissue of unknown cause. Its characteristic but not pathognomonic histopathological criteria include 1) non-caseating granulomas of the mammary lobule, 2) epithelioid cells, 3) multinucleated giant cells, 4) neutrophilic infiltration, and 5) frequent lymphocytes, eosinophils, and plasma cells. ${ }^{8} \mathrm{~A}$ priority of right diagnosis is infection exclusion, notably tuberculosis (TB) which also mimic idiopathic granulomatous mastitis [IGM.]

Idiopathic granulomatous mastitis [IGM] is an uncommon pathology with a documented predominance in Asian and Hispanic females. Literature evidence is mostly entails case reports and small series. Agreement on standardized treatment strategies is lacking in the literature. Treatment options ranges from simple observation to excision of the inflammatory mass.

Idiopathic granulomatous mastitis [IGM] is an uncommon benign breast disease. The present work studied 10 patients of proved idiopathic granulomatous mastitis [IGM]. Although Idiopathic granulomatous mastitis [IGM] was first described forty years ago, exact cause and pathogenesis of the disease is still obscure. Literature assumed possible link with lactation, contraceptives, prolactinemia, heavy smoking and Corynebacterium organism. ${ }^{9-11}$ Longer duration of the disease and higher severity in puerperium was suspected in a single study. ${ }^{12}$ Ninety percent of the patients in the present study were in the childbearing period and one patient affected in the breast feeding period. In the present study significant relationship was found with smoking, and/or Corynebacterium infection.

The important point in the diagnosis is to differentiate idiopathic granulomatous mastitis [IGM] from 
malignant breast. In our series, $70 \%$ of the patients presented with suspicious mass, and sixty percent were suspicious for cancer breast by radiography. Histopathological confirmation played prime role in the diagnosis. Idiopathic granulomatous mastitis [IGM] diagnosis is suspected by exclusion. Cultures and staining for micro-organisms (bacteria, fungi, and mycobacteria) must be performed especially in districts with TB prevalence.

Treatment of idiopathic granulomatous mastitis [IGM] is always challenging. No standard treatment postulated till now. In a multicenter retrospective study included more than seven hundred idiopathic granulomatous mastitis [IGM] patients. ${ }^{13}$ Fifty percent of the cases were treated with multimodality approaches followed by steroids in $40 \%$ of the cases, and surgery alone in $10 \%$ of the cases. Rate of recurrence was $17 \%$. Local excision (wide) with or without steroid therapy still the preferred treatment by many authors. ${ }^{14,} 15$ Excisional surgery showed best results as regard healing time. Delayed healing and high recurrence rate were reported $(12-48 \%$ and 10 $40 \%$, respectively). The present work showed less healing time following surgery. Surgical therapy had $30 \%$ wound morbidity and $10 \%$ recurrence rate. Despite high surgical morbidity, some surgeons advocate successful reconstruction following surgery. 16,17

Most authors recommended steroid containing protocols. DeHertogh and his colleagues were pioneers, they advocate steroid therapy for idiopathic granulomatous mastitis [IGM] with very good results. ${ }^{18}$ Other series published similar results. ${ }^{19,20}$ On the other hand Bani-Hani and associate said that steroid therapy may be associated with lower risk for breast deformity after surgical operations. ${ }^{21}$ Pandey and colleagues in their study reported that steroids were effective in fifty patients that managed from idiopathic granulomatous mastitis [IGM]. ${ }^{22}$

Surgical removal of the breast lump has been advocated in literature with accepted postoperative outcome. ${ }^{23-24}$ Other researchers announced infrequent recurrence even following mastectomy. ${ }^{25}$ Excision may be associated with ugly outcome and noticeable scarring, as idiopathic granulomatous mastitis [IGM] almost presented as a sizable mammary mass. ${ }^{21}$

Because of complications of steroid therapy and complications of surgery, many authors adopted conservative management of idiopathic granulomatous mastitis [IGM]. Recurrence as reported by Bouton et al. in $10 \%$ of their 27 patients treated conservatively (without steroid treatment and/or surgical excision) for a duration of 7.5 months to complete resolution. Sixteen cases needed other procedures as repeated percutaneous aspiration. ${ }^{26}$ Eight cases managed conservatively with Lai and associates. They said that 4 cases developed resolution (after 14.5 months of median duration). ${ }^{27}$

No worldwide consensus about the best line of treatment of idiopathic granulomatous mastitis [IGM]. Other authors recommended the use of immune- modulatory agents as methotrexate as a new drug therapy for idiopathic granulomatous mastitis [IGM]. Those trials contained series of $2-4$ patients. ${ }^{28,} 29$ Others advocated the use of combination of steroids and methotrexate. ${ }^{30-32}$

All cases with proven idiopathic granulomatous mastitis [IGM] treated with prednisolone $(20 \mathrm{mg}$ twice daily for 4 weeks, then the dose tapered over another 4 weeks), with percutaneous or open abscess drainage, plus culture and sensitivity of pus. Excision of the phlegmon was reserved as a final management in the present study. Smoking and presence of Corynebacterium in culture were identified as important risk factors for disease recurrence. Effect of smoking in non-lactational mastitis remains unknown. Association between non-lactational mastitis and smoking ${ }^{33}$ and results of present study augment this possible association.

Corynebacterium species (non-diphtheriae) are regarded as opportunistic bacteria (they rarely cause clinical infection in human body). It was first isolated from a sputum sample in 1998. ${ }^{34}$ It is a non-motile, Gram +ve, non-spore-forming, rod-shaped, nonpigmented organism with typical coryneform shape and isolated in human infections, notably in idiopathic granulomatous mastitis [IGM]. ${ }^{35,}{ }^{36}$ Recent study identified 46 strains of Corynebacterium from 41 breast-related specimens. As regard its slow-growing span, the importance of Corynebacterium have been under-estimated previously in the literature. ${ }^{34}$ Highly selective agar promote growth and isolation from cases infected with Corynebacterium. ${ }^{37}$

The present work has detected that Corynebacterium should be considered as an important prognostic factor for idiopathic granulomatous mastitis [IGM] recurrence. Therefore, meticulous and careful microbiological assessment is recommended for all inflammatory breast lesions suspicious of idiopathic granulomatous mastitis [IGM]. As regard to the limited samples of Corynebacterium in the present study, usefulness of antibiotic against Corynebacterium in this condition of idiopathic granulomatous mastitis [IGM] remains unclear.

\section{CONCLUSION}

Nowadays, idiopathic granulomatous mastitis (IGM) could be considered as a medical condition. It lost its surgical interest except for few cases of complicated or relapsing course. The main three aetiological explanations should be taken into account. Main treatment should be focused on three targets immune, and endocrine systems, and infectious organisms single or in combination. Pharmacological drugs ranged from immunomodulators to corticosteroids to drugs targeted endocrine system. Idiopathic granulomatous mastitis (IGM) has multidirectional and complicated therapeutic impact. It is identified as complex multi-factorial pathology that should concern different specialties in endocrinology, radiology, immunology, pathology, and bacteriology. Addressing the right cause, it may be possible for addressing the correct therapy. This 
results in avoidance of wrong decisions and unnecessary hospital admission and ineffective costs. This chronic inflammatory disease has recurrence incidence of $10 \%$ in the present study. There was history of smoking with Corynebacterium isolation from excised specimen.

\section{REFERENCES}

1. Michael C, Vincent $\mathrm{C}$ and Wei J. Idiopathic granulomatous mastitis: a 10-year study from a multicentre clinical database. Pathology. 2018; 1 6.

2. Prakasit $\mathrm{C}$, Panya $\mathrm{T}$, Chairat $\mathrm{S}$, et al. Idiopathic granulomatous mastitis: A retrospective cohort study between 44 patients with different treatment modalities. Ann Med Surg (Lond). 2018; 12(36): 162-7.

3. Fazzio RT, Shah SS and Sandhu SP. Idiopathic granulomatous mastitis: imaging update and review. Insights Imaging. 2016; 7:531-9.

4. Calis $\mathrm{H}$ and Karabeyoglu SM. Follow-up of granulomatous mastitis with monitoring versus surgery. Breast Dis. 2017; 37:69-72.

5. Nidhia $S$; and Mariloua C. Corynebacterium kroppenstedtii: a challenging culprit in breast abscesses and granulomatous mastitis. Current Opinion in Obstetrics and Gynecology. October 2019: 31(5); 325-32.

6. Lei X, Chen $\mathrm{K}$ and Zhu L. Treatments for idiopathic granulomatous mastitis: systematic review and metaanalysis. Breastfeed Med. 2017; 12: 415-21

7. Wolfrum A, Kümmel S, Theuerkauf I, et al Granulomatous Mastitis: A Therapeutic and Diagnostic Challenge. Breast Care. 2018; 13:413 -8 .

8. Néel A, Hello M, Cottereau A, et al. Long-term outcome in idiopathic granulomatous mastitis: a western multicentre study. QJM. 2013;106:43341.

9. Altintoprak F., Kivilcim T., Ozkan O.V. Aetiology of idiopathic granulomatous mastitis. World J Clin Cases. 2014; 2(12):852-8.

10. Hur S.M., Cho D.H., Lee S.K., et al. Experience of treatment of patients with granulomatous lobular mastitis. J. Korean Surg. Soc. 2013; 85(1):1-6.

11. Kayahan M., Kadioglu H and Muslumanoglu M. Management of patients with granulomatous mastitis: analysis of 31 cases. Breast Care (Basel). 2012; 7(3):226-30.

12. Shin Y.D., Park S.S., Song Y.J., et al. Is surgical excision necessary for the treatment of Granulomatous lobular mastitis?. BMC Wom. Health. 2017; 17(1): 49.

13. Uysal E., Soran A and Sezgin E. Granulomatous Mastitis Study G. Factors related to recurrence of idiopathic granulomatous mastitis: what do we learn from a multicentre study?. ANZ $J$. Surg. 2018; 88(6):635-9.

14. Yau F.M., Macadam S.A., Kuusk U, et al. The surgical management of granulomatous mastitis. Ann. Plast. Surg. 2010; 64(1):9-16.

15. Yabanoglu H., Colakoglu T., Belli S., et al. A comparative study of conservative versus surgical treatment protocols for 77 patients with idiopathic granulomatous mastitis. Breast J.2015; 21(4):363-9.

16. Hladik M., Schoeller T., Ensat F., et al. Idiopathic granulomatous mastitis: successful treatment by mastectomy and immediate breast reconstruction. J. Plast. Reconstr. Aesthetic Surg. 2011; 64(12):1604-7.

17. Yukawa M., Watatani M., Isono S., et al Management of granulomatous mastitis: a series of 13 patients who were evaluated for treatment without corticosteroids. Int. Surg. 2015; 100(5): 774-82.

18. DeHertogh DA, Rossof AH, Harris AA. Prednisone management of granulomatous mastitis. N Eng J Med. 1980; 308: 799-800

19. Gunduz Y, Altintoprak F and Tatli Ayhan L. Effect of topical steroid treatment on idiopathic granulomatous mastitis: clinical and radiologic evaluation. Breast J . 2014; 20: 586-91.

20. Altintoprak F, Kivilcim T and Yalkin O. Topical steroids are effective in the treatment of idiopathic granulomatous mastitis. World J Surg. 2015; 39: 2718-23.

21. Bani-Hani KE, Yaghan RJ and Matalka S. Idiopathic granulomatous mastitis: time to avoid unnecessary mastectomies. Breast J. 2004; 10: 318-22.

22. Pandey TS, Mackinnon JC and Bressler L. Idiopathic granulomatous mastitis-a prospective study of 49 women and treatment outcomes with steroid therapy. Breast J. 2014; 20: $258-66$.

23. Asoglu O, Ozmen V and Karanlik H. Feasibility of surgical management in patients with granulomatous mastitis. Breast J. 2005; 11: 10814.

24. Zhang X, Li Y, Zhou Y, et al. A systematic surgical approach for the treatment of idiopathic granulomatous mastitis: a case series. Gland Surg. 2020; 9(2):261-70.

25. Taghizadeh R, Shelley OP, Chew BK. Idiopathic granulomatous mastitis: surgery, treatment, and reconstruction. Breast J. 2007; 13: 509-13.

26. Bouton BE, Jayaram L and O'Neill PJ. Management of idiopathic granulomatous mastitis with observation. Am J Surg. 2015; 210: 258-62.

27. Lai ECH, Chan WC and Ma TK. The role of conservative treatment in idiopathic granulomatous mastitis. Breast J. 2005; 11: 4546.

28. Schmajuk G and Genovese MC. First report of idiopathic granulomatous mastitis treated with methotrexate monotherapy. J Rheumatol. 2009; 36: 1559 .

29. Akbulut S, Arikanoglu $\mathrm{Z}$ and Senol A. Is methotrexate an acceptable treatment in the management of idiopathic granulomatous mastitis? Arch Gynecol Obstet. 2011; 284: 118995.

30. Wilson J, Massoll $\mathrm{N}$ and Marshall J. Idiopathic granulomatous mastitis: in search of a therapeutic paradigm. Am Surg. 2007; 73: 798 802 .

31. Sheybani F, Sarvghad M and Naderi HR. Treatment for and clinical characteristics of 
granulomatous mastitis. Obstet Gynecol. 2015; 125: 801-7.

32. Skandarajah A and Marley L. Idiopathic granulomatous mastitis: a medical or surgical disease of the breast?. ANZ J Surg. 2015; 85: 979-82.

33. Dixon JM, Ravisekar O and Chetty U. Periductal mastitis and duct ectasia: different conditions with different aetiologies. Br J Surg. 1996; 83:820.

34. Collins MD, Falsen E and Akervall E. Corynebacterium kroppenstedtii sp. nov., a novel Corynebacterium that does not contain mycolic acids. Int J Syst Bacteriol, 1998; 48: 1449-54.

35. Riegel $\mathrm{P}$, Liegeois $\mathrm{P}$ and Chenard MP. Isolations of Corynebacterium kroppenstedtii from a breast abscess. Int J Med Microbiol. 2004; 294: 413-6.

36. Tauch A, Schneider J, Szczepanowski R. Ultrafast pyrosequencing of Corynebacterium kroppenstedtii DSM44385 revealed insights into the physiology of a lipophilic Corynebacterium that lacks mycolic acids. J Biotechnol.2008; 136: 22-30.

37. Wong SCY, Poon RWS and Foo CH. Novel selective medium for the isolation of Corynebacterium kroppenstedtii from heavily colonized clinical specimens. J Clin Pathol. 2018; 71: 781-6. 